\title{
A Kind of CTA Bone Removal Technology Based on the Improved Watershed Algorithm
}

\author{
Tan Jianhao ${ }^{1}$, Zhang Jing ${ }^{1} \&$ Wang $\mathrm{Ya}^{1}$ \\ ${ }^{1}$ Electrical and Information Engineering College, Hunan University, Changsha, China \\ Correspondence: Tan Jianhao, Electrical and Information Engineering College, Hunan University, Changsha, \\ Hunan 410082, China. Tel: 86-135-0848-7511. E-mail: tanjianhao96@sina.com.cn
}

Received: July 6, 2012 Accepted: July 23, 2012 Online Published: August 17, 2012

doi:10.5539/cis.v5n5p81 URL: http://dx.doi.org/10.5539/cis.v5n5p81

\begin{abstract}
Based on analyzing the drawbacks existing in traditional watershed algorithms for bone removal from CTA (Computed Tomography Angiography, CTA), this paper presents an improved interactive watershed algorithm. The improved watershed algorithm is based on sorting and graded overflow of fast watershed algorithms, and the merging process of catchment basins is intervened by a merging threshold which is given by users so as to take an effect on segmentation results. The algorithm can record such basic information as the labels and ridge points of each basin in graded overflow basin marking, set merging threshold by means of user interaction for controlling merging process effectively, and suppresses over segmentation. At last, the improved algorithm is applied to bone removal from CTA Images, and three-dimensional rendering is taken for CTA Images after bone removal. The experimental results indicate that the improved algorithm prevents over segmentation in watershed transformation effectively and removes bone structure accurately.
\end{abstract}

Keywords: image segmentation, hierarchical, mathematical morphology, improved watershed algorithm, catchment basin, CTA, bone removal

\section{Introduction}

Medical image processing uses image segmentation to extract lesion area from a very complex background image for further analysis and processing, and the complexity of medical images makes medical image segmentation become a research hotspot and difficulty in medical image processing. One of the most challenging problems is how to remove bone structure effectively from floods of Computerized Tomography Angiography (CTA) images so as to obtain vascular and internal organ information which is used to diagnose illness (Yongbum et al., 2005).

Because vascular is of the three-dimensional tree structure, carrying on visualization diagnosis in two-dimensional space is an extremely difficult matter. Therefore, reappearing vascular structure by three-dimensional visualization becomes very important and essential. Maximum Intensity projection (MIp) is one of the most commonly-used methods, but the high-brightness bone structure in CTA is the main obstacle in reappearing vascular structure by three dimensional visualization, so bone removal is necessary firstly (Abdalmajeid, 2001). The CT value of bone in CTA image is not in a constant range, and bone structure and vascular structure overlap in the brightness distribution of images, which makes bone removal from CTA more difficult (Yongbum et al., 2005). As shown in Figure 1, bone, soft tissue, fat and air gray levels have their respective scopes and overlap partly (Robert, Drebin, Loren, \& Pat, 1988).

Bone removal from CTA is a typical application in medical image segmentation area whose main problem is that the local characteristic of bone in images is very strong, and bone gray level is widely distributed to different parts. What's more, the boundary of different organizations is blurry, so the commonly used segmentation algorithms based on global optimization have difficulty in effective segmentation. The watershed algorithm based on mathematical morphology is an effective segmentation method which can accurately locate the edges of images. Besides, the obtained points in small basins are of strong local consistency. However, there is usually a serious segmentation. Therefore, how to overcome that has been a central issue.

An interactive watershed algorithm is presented in this paper. The algorithm can record such basic information as the labels and ridge points of each basin in graded overflow basin marking, set merging threshold by user 
interaction for controlling merging process effectively, and suppresses over segmentation. The experimental results demonstrate the effectiveness of the improved algorithm.

\section{Traditional Watershed Algorithms}

The watershed algorithm (Roerdink \& Meijster, 2001) is an image segmentation method based on mathematical morphology built on topology theory and its basic thought is originated from geography where images are looked as topological geomorphology on geodesy. The pixel of each point in images represents its altitude, and every local minimum value and its influence zone is called a catchment basin whose boundary is known as a watershed.

There are many ways to realize watershed transformation including recipitation simulation and immersion simulation, etc. (Roerdink \& Meijster, 2001). The immersion simulation method given by Vincent is very representative of other proposed ways (Vincent \& Soille, 1991). The method simulates this process: firstly a small hole is pierced at the bottom of each local minimum value, then the image model is immersed gradually into water and the local minimum value influence zone spreads around by degrees, as a result of the immersion, a peak line called watershed is produced at the place where the two basins meet. These basic conceptions are shown in Figure 2.
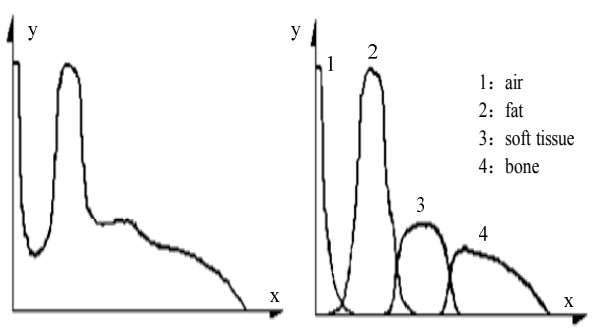

Figure 1. Original histogram and material distribution

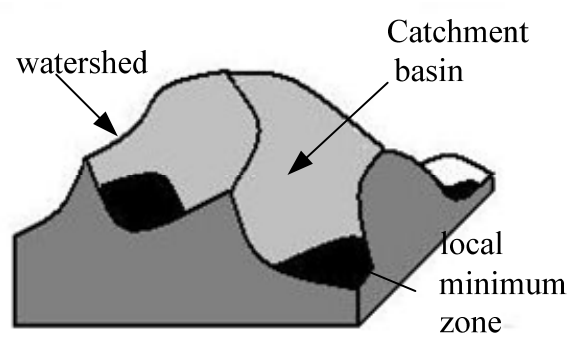

Figure 2. Local minimum zone, catchment basin and watershed of gray level Images

\subsection{Advantages of the Traditional Watershed Algorithm}

Many advantages of the watershed algorithm can be easily seen. Firstly, continuous edges of one-pixel width are obtained; what's more, it can deal with two or three, even $n$, dimensional images and graphs; eventually, several objects are able to be segmented at the same time and be labeled easily. Therefore, the watershed algorithm is sharply developed and extensively applied to medical image segmentation. It has become a powerful tool with which doctors change qualitative analysis on images into quantitative one (Zhang, Yao-Yong, \& Liu, 2008).

\subsection{Existing Drawbacks of the Traditional Watershed Algorithm}

The watershed algorithm is sensitive to the change of gray level, noise or other slight gray level changes in images will generate over segmentation. Two segmentation results of CTA slice images are shown in Figure 3. Figure 3(a) introduces the result of watershed segmentation based on gradient, and Figure 3(b) gives the one of common watershed segmentation after smooth gradient processing.

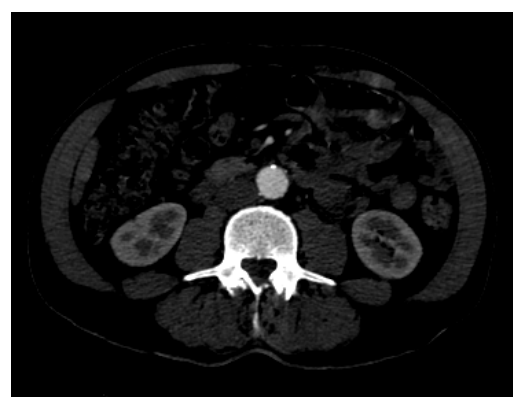

(a)

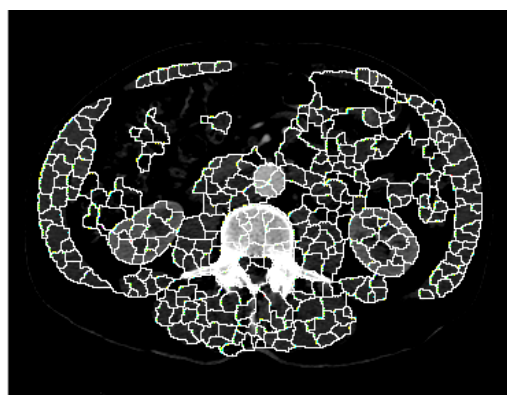

(b)

Figure 3. Two segmentation results of CTA slice images (a) watershed segmentation based on gradient; (b) common watershed segmentation after smooth gradient processing 
It can be seen from Figure 3 that image bones are divided into many small catchment basins due to over segmentation. And because of small area number of pixels in each basin, it is difficult to make sure of what tissue the basin belongs to according to the property of points in some basin zone. So, how to control over segmentation is always the main problem of the watershed segmentation algorithm.

\section{Improved Interactive Watershed Algorithm}

\subsection{Basic Ideas of the Improved Algorithm}

The improved interactive watershed algorithm based on morphology is adopted in this paper (Hahn \& Peitgen, 2003). The basic idea of the algorithm is: firstly, bone-voxels and nonbone-voxels are controlled through global thresholds $\mathrm{T}_{1}$ and $\mathrm{T}_{2}$ (Roerdink \& Meijster, 2001), and then the interactive watershed operations are applied to fuzzy voxels. The interactive watershed algorithm based on immersion simulation built on quick watershed adopts tree structure to organize catchment basins. A local minimum value represents an original basin on the same level. After finishing basin marking, basin labels and another interactive input global parameter $h_{p f}$ are used to control the process of basin mergence.

\subsection{Graded Overflow Basin Marking}

The watershed algorithm proposed by Vincent is a classical regional marking method which is built on the concept of geodesic distance and the pixel basin labels are determined by calculating influence zones. However, the correlation between the image pixels is more important than pixels themselves in image segmentation.

The interactive algorithm basin marking emphasizes on the connectivity among pixels, and the use of hierarchical order immersion marking can not only decrease unnecessary distance calculation time but also reduce running time to a certain extent compared to the method to ascertain basin boundaries by calculating influence zones. Besides, it is able to improve segmentation accuracy because of adopting spatial domain voxels.

The interactive watershed algorithm which is built on fast watershed transformation gets the catchment basin hierarchical expressive form called a kind of tree-like structure tag image data by means of submerging $n+1$ dimensional topography. A case of one dimension is represented in Figure 4. The "terrain" immersion is simulated from low to high. A ridge point $m_{1}$ is obtained at gray level $g_{f}=33$, and recorded as an item that is going to be merged, and the seed points of the neighboring basins are $b_{1}$ and $b_{2}$ respectively.

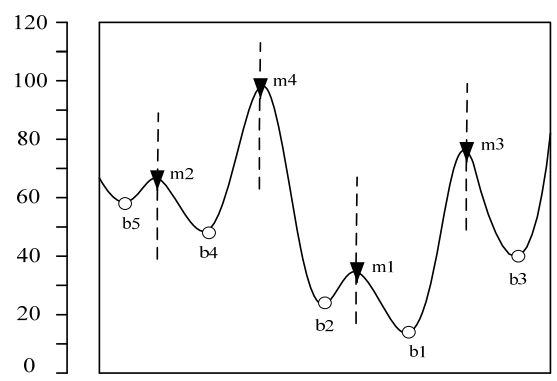

Figure 4. Watershed Immersion Simulation Process

Figure 4 indicates that the number of catchment basins and ridge points increase with the argument of overflow height $\mathrm{g}_{\mathrm{f}}$, and eventually a kind of tree-like structure data are obtained as is illustrated in Figure 5 which records mutual position relationship among voxels, for example, the basin information $\mathrm{B}$ and the information $\mathrm{M}$ of the items to be merged in Table 1.

Table 1. Data structure obtainable by the end of hierarchical immersion

\begin{tabular}{cc|cccc}
\hline \multicolumn{2}{c|}{ B } & \multicolumn{4}{|c}{$\mathbf{M}$} \\
\hline$\beta$ & $g$ & $\mu$ & $g$ & $k$ & $\alpha$ \\
1 & 15 & 1 & 33 & 2 & 1 \\
2 & 22 & 2 & 68 & 5 & 4 \\
3 & 40 & 3 & 76 & 3 & 1 \\
4 & 48 & 4 & 98 & 4 & 2 \\
5 & 60 & & & & \\
\hline
\end{tabular}


In the process of graded overflow basin marking, each pixel in a sorted image could be accessed as the increment of pixel gray level values. The process begins at a very small area, and operates gradually according to gray level values. If the neighboring ones of some point to be processed are all unmarked, the point is taken as a new basin seed point so as to produce a new basin. Otherwise, the label of the deepest basin in the linking area of the point is marked, and record the around basin information as an advantage of merging calculation on catchment basins. The immersion simulation ends when the immersion height reaches the maximal gray level value.

A kind of tree structure data described in Figure 5 and Figure 6 are acquired by graded overflow marking. In the original basin seed information $\mathrm{B}$, the gray level $\mathrm{g}$ of basin seeds (local minimum value points) and the sorted primitive index label $\beta$ are recorded, while in the related information $M$ about items to be merged, the information g about ridge points, where $\mu$ stands for a ridge index label, and $\mathrm{k}[\mu]$ and $\alpha[\mu]$ are the index label $\beta$ of seed points in the basin information $B$ in the neighboring basin of corresponding ridge points, are noted.

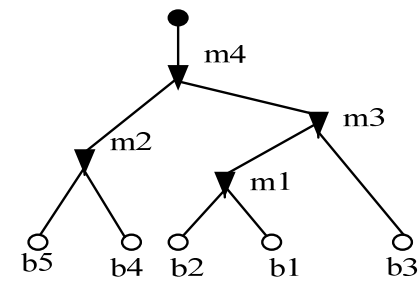

Figure 5. Tree structure data obtainable by overflow

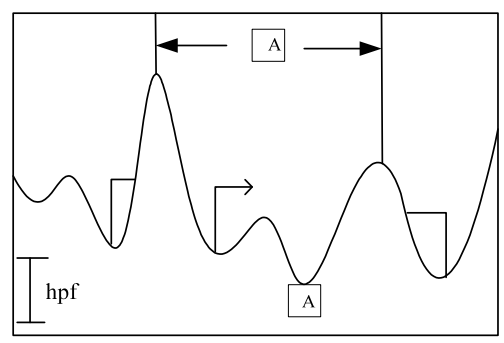

Figure 6. Schematic diagram of catchment basins mergence

\subsection{Catchment Basin Mergence}

The improved watershed algorithm lies in catchment basin mergence. It combines one basin with another through basin marking and merging threshold $\mathrm{h}_{\mathrm{pf}}$ set by users. In the multi-scale information processing of images, the relative potential energy and the gaps among points are the indicators of watershed operations (Zhang, Yao-Yong, \& Liu, 2008), and the smaller the gaps among points are, the lower the importance of watershed is. Basin depth is used to define the merging process. As shown in Figure 6, given merging threshold $\mathrm{h}_{\mathrm{pf}}$ and basin marking A (basin seed points), if the adjacent basin depth is smaller than the threshold, the basin expansion is taken until the new adjacent basin depth becomes greater than the threshold.

The main function of user interactive operations in catchment basin mergence is to determine the threshold $\mathrm{h}_{\mathrm{pf}}$, and operators intervene basin merging process manually by changing the threshold $\mathrm{h}_{\mathrm{pf}}$. In the initialization of the algorithm, such relative data information as basin labels corresponding to image elements obtained by immersion process are fixed down via ascertaining global threshold $\mathrm{T}_{1}$ and $\mathrm{T}_{2}$. So, sorting and immersing once again is not needed when intervening basin mergence by changing the threshold $h_{p f}$ so that the program running time is decreased. As a result, operators are able to adjust $h_{p f}$ intuitively in the situation that they can observe the results so as to get new segmentation outcomes. What's more, the process just costs a little time and can prevent over segmentation to the almost extent.

\section{Application of the Improved Algorithm to Bone Removal from CTA Images}

\subsection{Effects of Merging Thresholds on Segmentation Results}

The boundary between bone and vascular would become more blurry due to the influence of such factors as partial volume effect, etc. Besides, the vascular tissue gray-levels, which are to a certain extent unpredictable, are related to dosage and testing time. Therefore, it is necessary that a larger initial threshold $\mathrm{T}_{1}$ and a lower limit threshold $\mathrm{T}_{2}$ be selected properly in watershed bone removal to make sure that all voxels whose gray-levels are greater than $T_{1}$ are bone voxels and the voxels whose gray-levels range from $T_{1}$ to $T_{2}$ are fuzzy voxels which may be bone voxels or not, while the others are doubtlessly not bone voxels (Wu, Hou, Zhang, Qin, \& Ou, 2003) Classifying fuzzy voxels should be lay emphasis on more than certain bone voxels and unbone ones when undertaking image segmentation.

Generally speaking, CT values $\left(\mathrm{T}_{1}\right)$ of bone or calcified vascular tissues in CTA images are greater than 576, while ones $\left(\mathrm{T}_{2}\right)$ of organ tissues in human bodies, unnecessary to be segmented, are smaller than 176. Certainly, the choice of $T_{1}$ and $T_{2}$ can be determined by manual interactive mode and other ineffective classification methods (say the method of OTSU), which is not discussed once again here. The pre-segmentation of images is 
capable of reducing the number of pixels which need be manipulated in the image and improving segment accuracy effectively. At the same time, it decreases segmentation time.

Considering a specific experiment, all the points whose gray-levels are greater than $\mathrm{T}_{1}$ are set as bone seeds, and then watershed processing is applied to points whose gray-levels are greater than $T_{2}$. The sizes of merging basins are able to be changed by means of adjusting HPF. Finally, it could be made sure whether the tissues are bone or calcified vascular ones via the number of bone seeds in each basin.

The bone removal operations of belly CTA images are presented in this paper. Given $T_{1}=576$, and $T_{2}=176$, $h_{p f}$ is gradually increased by the interactive way. Two different segmentation results gained with different $h_{p f}$ values by choosing two slices in original images are illustrated in Figure 7 and Figure 8, where the values of $\mathrm{h}_{\mathrm{pf}}$ are 1000, 2000 and 3000, respectively. VC 2008 is applied to programming in this procedure. Dealing time can be controlled within 20 s for $512 * 512 * 200$ layers of images. Furthermore, when the operators feel not satisfied with the initial segmentation result, they could track the new bone removal results quickly by adjusting $\mathrm{h}_{\mathrm{pf}}$ manually, which just costs 3s. Figure 7 indicates that bone, soft tissue, fat and air, etc have their own gray levels in the two-dimensional slice images. Figure 8 shows that merging thresholds have an effect on segmentation results in two-dimensional slice images.

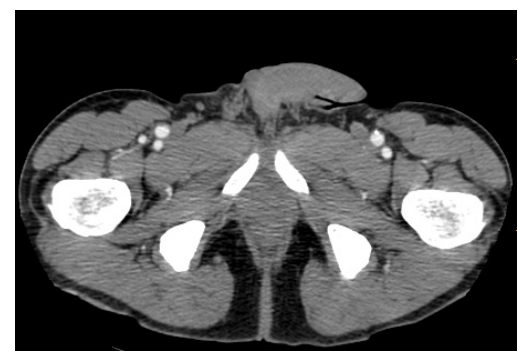

(a)

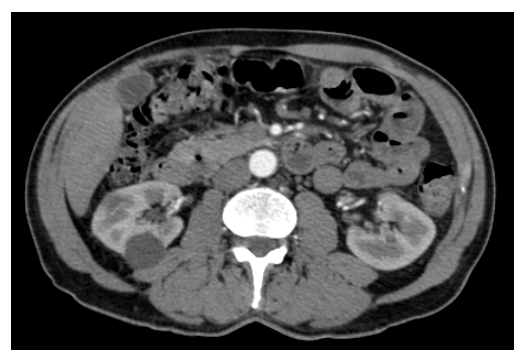

(b)

Figure 7. CTA slice image (a) image of the 32th slice; (b) image of the 197th slice
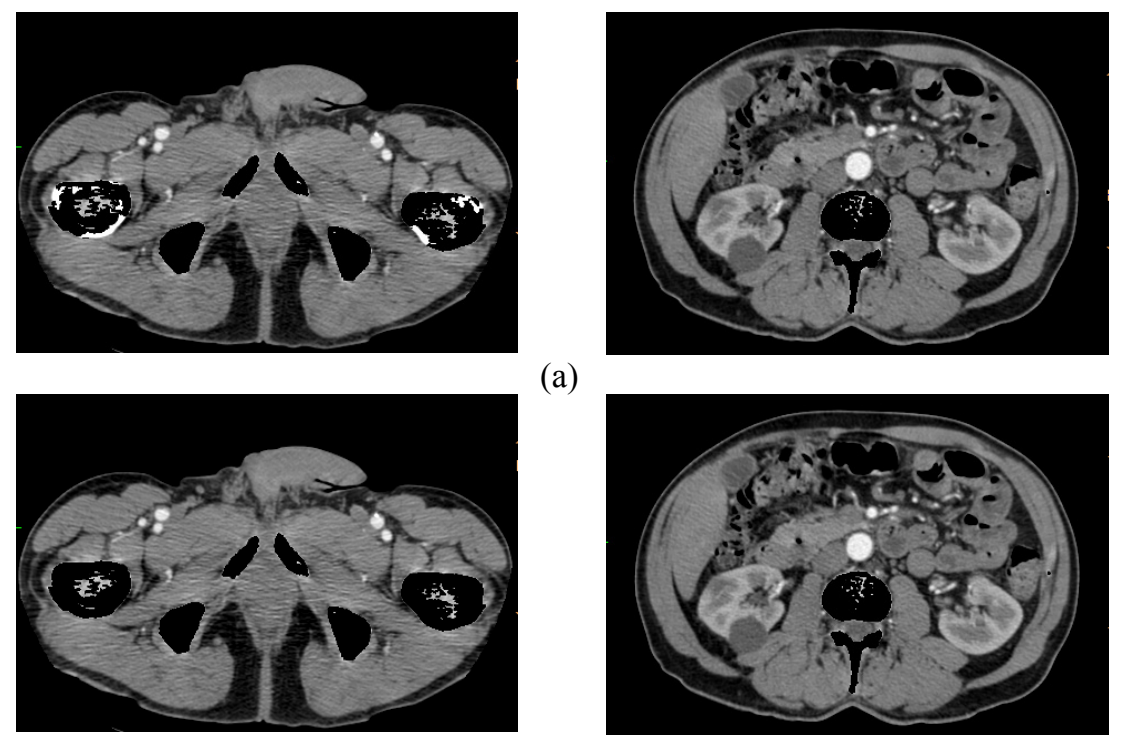

(a)

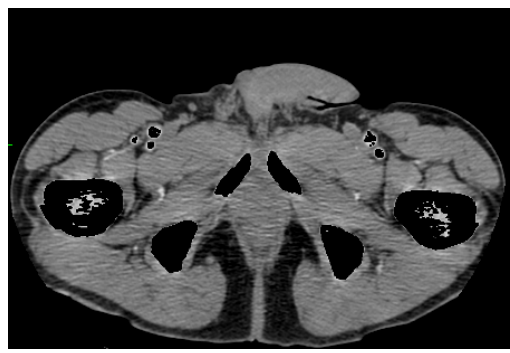

(b)
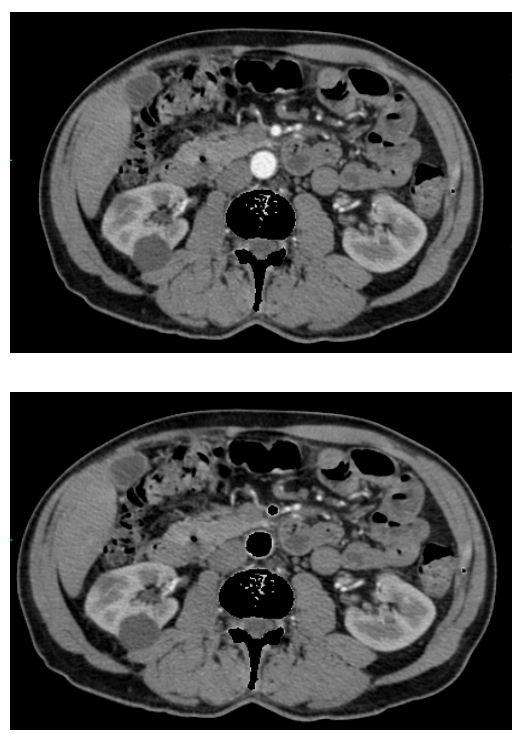

(c)

Figure 8. Slice segmentation results (a) $h_{p f}=1000 ;$ (b) $h_{p f}=2000 ;$ (c) $h_{p f}=3000$ 


\subsection{3-D Rendering of CTA Images after Bone Removal}

If three-dimensional rendering is taken for above CTA Images after Bone Removal respectively, The results are shown in Figure 9. Where the three-dimensional imaging of original CTA images is indicated in Figure 9(a) where it can be seen that the vascular structure is surrounded with the bone structure. Figure 9(b) demonstrates that when merging threshold $\mathrm{h}_{\mathrm{pf}}$ is smaller, the less the bone structure removed by segmentation is, the more the kept bone one is more. It can be concluded from Figure 9(c) that as threshold $h_{p f}$ grows, the removed bone structure increases so that the segment result is improved. Figure 9 (d) shows that with the increment of $h_{p f}$ once more, a part of vascular structure is removed so that the segment result is gradually becoming bad.

The experiment illustrates that, in executing the algorithm, the operator is capable of intervening segmentation results by changing threshold $\mathrm{h}_{\mathrm{pf}}$ according to practical demands.

The vascular structure of CTA images after bone removal can be seen from Figure 9 clearly, which indicates the improved algorithm can remove the bone structure in CTA images effectively.

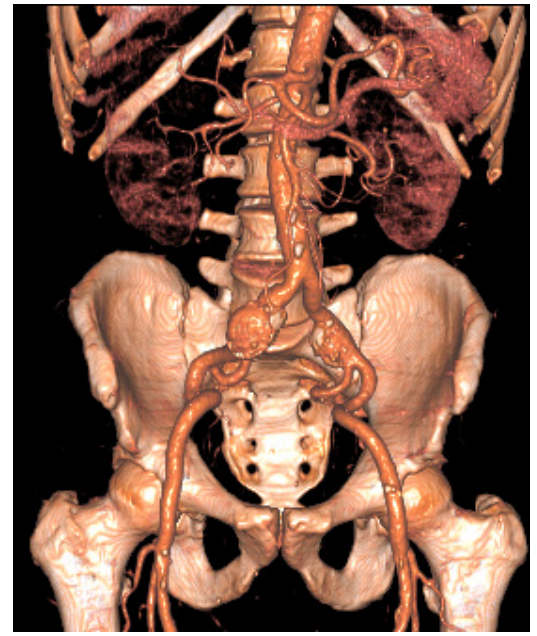

(a)

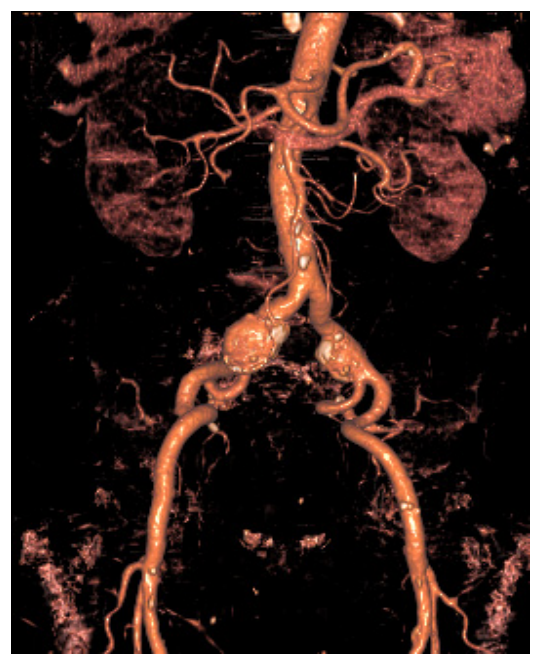

(c)

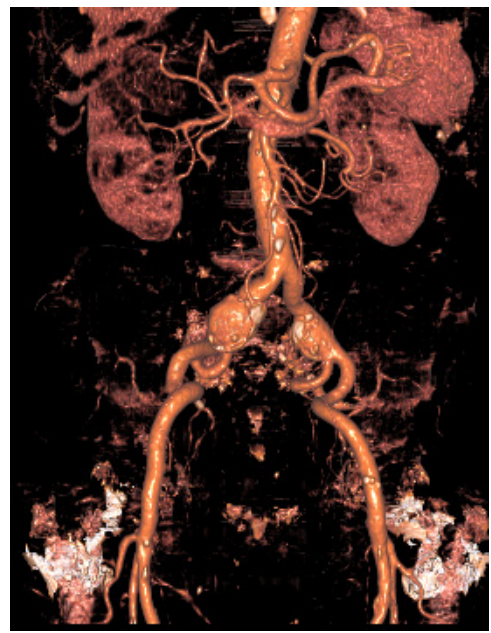

(b)

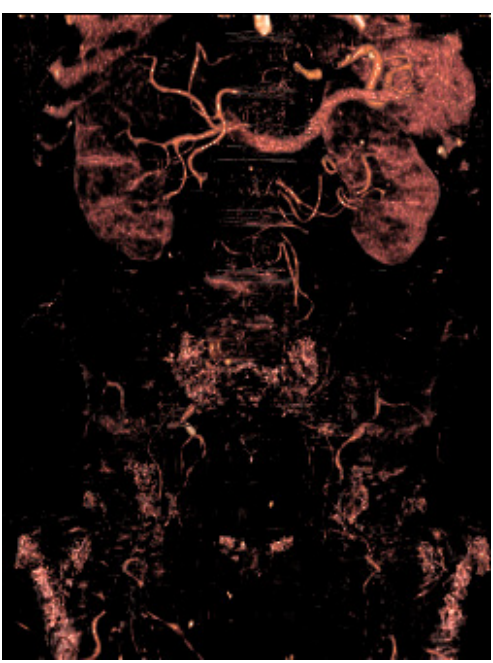

(d)

Figure 9. 3-D reconstruction original images and 3-D rendering of the images after bone removal

\section{Conclusions}

In the paper, the improved interactive watershed algorithm is applied to bone removal from CTA images, and three-dimensional rendering is taken for CTA Images after bone removal. The experimental results indicate that the improved algorithm is able to remove bone structure in CTA images effectively. What's more, because bone removal can be realized very intuitively by the manual interactive method, over segmentation existing in 
traditional watershed algorithms can be avoided. Therefore, the application of the improved watershed algorithm after appropriate preprocessing for CTA images provides a better service and reference for clinical medicine.

\section{References}

Abdalmajeid, M. A., \& Gopal, B. A. (2001). Semi-automatic Bone Removal Technique from CT Angiography Data. 4322 Proceedings of SPIE, 4322, 1273-1283.

Hahn, K., \& Peitgen, H. (2003). IWT-Interactive Watershed Transform: A Hierarchical Method for Efficient Interactive and Automated Segmentation of Multidimensional Grayscale Images. Proc. Medical Imaging, SPIE 5032, San Diego, 12, 643-653.

Roerdink, J., \& Meijster, A. (2001). The Watershed Transform: Definitions, Algorithms and Parallelization Strategies. Fundamenta Informaticae, 41, 187-228.

Robert, A., Drebin, Loren, C., \& Pat, H. (1988). Volume Rendering. Computer Graphics, 22(4), 65-74. http://dx.doi.org/10.1145/378456.378484

Vincent, L., \& Soille, P. (1991). Watersheds in Digital Space: An Efficient Algorithm Based on Immersion Simulation. IEEE Trans. Pattern Anal. Machine Intell, 13(6), 583-598. http://dx.doi.org/10.1109/34.87344

Wu, L., Hou, J., Zhang, Y., Qin, X., \& Ou, Z. (2003). Bone Removal from CT Images by Neighbor Operation. Chinese Journal of Biomedical Engineering, 22(23), 199-202.

Yongbum, L., Du-Yih, T., Satomi, I., Ryujiro, Y., Takeshi, H., Hiroshi, F., ... Hiroaki, H. (2005). An Automated Bone Removal Technique for Extraction of Cerebral Vessels from Head CT Angiography. Proceedings of the IASTED International Conference, BIOMEDICAL ENGINEERING, Innsbruck, Austria, 12, 16-18.

Zhang L., Yao, Y., \& Liu, Z. (2008). A Comparative Potential Energy Watershed Algorithm. Journal of Xidian University (Natural Science), 35(1), 121-124. 